\title{
DPS COMPLEXOS EM ESTRUTURAS DE “CONSTRUAL"1
}

\section{Complex DPS in construal structures}

\author{
Mary Kato*
}

\begin{abstract}
RESUMO
Em seu texto clássico de 1977, Chomsky distingue cadeias formadas por movimento - topicalização - de cadeias formadas sem movimento - deslocamento à esquerda. O motivação central para essa distinção era a existência de efeitos de ilha nas primeiras e sua inexistência nas últimas. Mais recentemente, alguns gerativistas estão tentando reduzir todas as relações de construal a movimento (Kayne, 2002; Hornstein, 2000; entre outros). O que eles propõem é que a derivação começa com uma estrutura contendo um DP complexo, no qual a cabeça e a cauda da cadeia aparecem inicialmente juntas e são separadas durante a derivação. Neste artigo, pretendo mostrar dois casos distintos em português brasileiro de estruturas de construal em que a derivação é facilitada se se assume a proposta do DP complexo. O primeiro caso tem relação com topicalização e deslocamento à esquerda com clíticos (CLLD) e o segundo caso, com o problema dos efeitos de conectividade em sentenças clivadas (cf. Boškovič 1997).
\end{abstract}

Palavras-chave: topicalização; deslocamento à esquerda; CLLD; clivadas; efeitos de conectividade; cadeias.

\begin{abstract}
In his (1977) classic work, Chomsky distinguished between a chain formed by movement (i.e. topicalization), and a chain formed without movement (i.e. left dislocation). The main motivation for this distinction was the existence of island effects in the former, and their lack in the latter. More recently some generativists are
\end{abstract}

${ }^{1}$ O presente trabalho teve o suporte do CNPq, Processo 301219/2008, e do Projeto Temático FAPESP 2006/00965-2. Agradeço à audiência da ANPOLL, 2010, em particular a Marina Augusto, minha debatedora, e a Carlos Mioto e Paulo Medeiros, pela leitura cuidadosa da versão preliminar do texto. Os problemas residuais são de minha inteira responsabilidade.

* Unicamp 
trying to reduce all construal relations to movement (Kayne, 2002; Hornstein 2000; among others). What they propose is that the derivation starts with a structure containing a complex DP, in which the head and the tail of the chain appear initially together to be separated during the derivation. In the present paper I intend to show two distinct cases of Brazilian Portuguese construal structures in which the derivation is facilitated if the complex DP proposal is assumed. The first case has to do with Topicalization and Clitic left Dislocation (CLLD) and the second case with the problem of connectivity effects in cleft sentences (cf. Boškovič 1997).

Keywords: topicalization; left dislocation; CLLD; clefts; connectivity effects, chains.

\section{INTRODUÇão}

No trabalho clássico de (1977), Chomsky distinguia uma cadeia formada por movimento-wh e uma cadeia formada sem movimento, em uma estrutura de Deslocamento à Esquerda $(\mathrm{DE})^{2}$.
a. This book ${ }_{i}$, you should read $t_{i}$.
b. As for this book $i$, you should read it $t$.

A principal motivação para essa distinção era a existência de efeito de ilhas na primeira e a ausência deste efeito na segunda.
a. ${ }^{*}$ This book $_{i}$, I like the journalist who reviewed ec ${ }_{i}$
b. As for this book ${ }_{i}$, like the journalist who reviewed $i_{i}$.

$\mathrm{Na}$ fase minimalista, alguns autores procuram reduzir todas as relações de "construal" a movimento (Kayne 2001, Hornstein 2000, entre outros). Para isso, as derivações começam com uma estrutura de DP

2 O exemplo mais normal de DE seria sem a cabeça as for, usualmente denominado Tópico Pendente. Tanto o caso de DE como de Tópico Pendente teriam o resumptivo pronominal no Português Brasileiro (PB), enquanto no Português Europeu (PE) essas construções se traduziriam como Deslocamento Clítico.

(i) (Quanto a) esse livro, eu gosto do jornalista que resenhou ele.

(ii) (Quanto a) esse livro, eu gosto do jornalista que o resenhou. 
complexo na qual a cabeça e a cauda da cadeia aparecem juntas para serem separadas durante a derivação. Em Kato e Raposo (2007), os autores distinguiram dois tipos de movimento: a) movimento integral, que resulta em topicalização, e b) movimento parcial, que dá lugar a deslocamento clítico ou deslocamento à esquerda, em que apenas parte de um DP complexo se move.

O que pretendo mostrar neste estudo é que uma teoria dos DPs complexos, de onde podem ser feitas extrações parciais, pode dar soluções interessantes a problemas distintos de subjacência e de conectividade ${ }^{3}$. A parte descritiva pormenorizada está em Kato e Raposo (2007), para o caso do Deslocamento Clítico, e em Kato e Mioto (2011), para o caso dos problemas de conectividade em pseudo-clivadas. Mas o que se extrai deste artigo é a validade de uma proposta teórica inovadora, que pode ser útil na resolução de outros problemas da gramática do português.

\section{Topicalização E DESLOCAMENTO CLítico}

No caso de "Clitic Doubling" (CLDOUBL), Uriagereka (1995) e Kayne (2001) assumem que o clítico e o DP que ele dobra são gerados como um constituinte complexo único, como em (4).

$$
\text { Le doy un libro a Juan. }
$$

(CLDOUBL)
a. Doy un libro [Juan le]
b. [Juan le] $]_{i}$ doy un libro $t_{i}$
c. Juan ${ }_{j}\left[t_{j} l e\right]_{i}$ doy un libro $t_{i}$
d. a Juan ${ }_{j}\left[t_{j} l e\right]_{i}$ doy un libro $t_{i}$ "merge" da preposição a
e. $\left[\left[t_{j} l e\right]_{i} \text { doy un libro } t_{j}\right]_{k}$ a Juan $t_{k}$

Derivação semelhante pode ser proposta para CLLD (Deslocamento Clítico à Esquerda), excetuando-se a última etapa da derivação:

3 o problema de conectividade, a ser detalhado na seção 3, tem a ver com as pseudoclivadas, nas quais muitas vezes há aspectos de ligação sem c-comando na sintaxe visível:

(i) Quem o João machucou foi a si mesmo. 
(5) A Juan le doy um libro.

Contudo, se todas as relações de "construal" são derivadas de movimento, é preciso encontrar explicações para dois problemas no Português:

a) por que em PE deslocamento clítico tem efeito de ilha quando o elemento tópico é um PP, mas é possível a partir de ilhas se o tópico é um DP, problema levantado por Raposo (1996), mas não respondido naquele trabalho?

a. O capitão ${ }_{i}$, encontrei [o negociante que lhe $_{i}$ telefonou ontem] $\checkmark \mathrm{EP}$

b. Ao capitão, encontrei o negociante que lhe $e_{i}$ telefonou ontem. $\quad * \mathrm{EP}$

b) por que topicalização em Português Europeu (PE) tem efeito de ilha (Raposo, 1986), mas não no Português Brasileiro (PB) (Galves, 1989)?
A: Onde é que vocês viram uma loja que vendia os CDs dos Beatles?
B: Os CDs dos Beetles, (nós) encontramos uma loja que vendia ec no aeroporto.
*EP • BP (Kato e Raposo, 2007)

Para resolver esses problemas, Kato e Raposo assumiram: a) que movimento integral tem restrições de subjacência, mas b) que efeitos de ilha podem ser também uma conseqüência de concordância de Caso, quando há movimento parcial, seguindo proposta de Boeck (2001). Note-se que em (6)'a temos discordância de caso entre o capitão e lhe e a sentença é boa, mas em (6)'b temos concordância de caso e a sentença exibe efeito de subjacência.

a. $O$ capitão nom default encontrei o negociante que lhe $e_{\text {dat }}$ telefonou ontem

b. *Ao capitão ${ }_{\text {dat }}$ encontrei o negociante que lhe dati $_{\text {telefonou }}$ ontem

Em relação ao problema (a), a hipótese de Cinque (1990) de que 
Topicalização e CLLD são sujeitas a ilhas, mas LD não, não se sustenta para o PE.

No italiano, a cabeça da cadeia é sempre um PP em CLLD, donde se explica por que nela sempre temos efeito de ilha.
Al mare, ci siamo già stati.
no mar lá somos já estados
'No mar, lá já estivemos.'

Para a segunda questão, Kato e Raposo propõem que no caso do PE temos movimento integral em (7)'(i), enquanto no PB o que temos é movimento parcial, a partir de um DP complexo, com um núcleo ocupado por um clítico nulo ${ }^{4}(7)^{\prime}$ (ii). Ora, movimento integral produz efeito de ilha independentemente de concordância ou discordância de Caso, enquanto, no caso de movimento parcial, a discordância de Caso é condição para a isenção a efeitos de ilha, que é o que temos no PB.

(7)' (i) *(Os CDs dos Beetles) $)_{i}$ vimos uma loja que vendia ec $\boldsymbol{i}_{\boldsymbol{i}}$ no aeroporto.

$\checkmark \mathrm{PE}$

(ii) (Os CDs dos Beetles) nom def vimos uma loja que vendia [ec i $_{\boldsymbol{i}}$ $[\varnothing]]_{\text {acus }}$ no aeroporto

$\checkmark \mathrm{PB}$

No trabalho de Kato e Raposo (2007), mostra-se ainda que nem sempre há contraste entre o PE e o PB, nos casos de topicalização. As duas variedades têm o mesmo comportamento quando há topicalização contrastiva, isto é, em nenhuma delas há sensibilidade a ilhas. Veja a resposta a seguir, aceitável tanto no PE quanto no PB:
A: Vocês encontraram uma loja que vendia CDs clássicos?
B: CDs clássicos antigos, (nós)encontramos uma loja que vendia ec no Iguatemi, mas CDs clássicos modernos, não $\checkmark \mathrm{EP} \checkmark \mathrm{BP}$

\footnotetext{
${ }^{4}$ Kato (1993) já havia proposto que objeto nulo seria um clítico nulo de 3 a pessoa.
} 
Para Kato e Raposo, o motivo dessa insensibilidade a ilhas no caso de topicalização contrativa tem a ver com o fato do movimento não se dar com um argumento, mas com topicalização residual de VP. Note-se que se a cópia do V não é apagada (o que é possível no português), a resposta é igualmente boa, isso porque topicalização de VP não é sensível a ilha, como foi descrito por Matos (1992).

(10) Encontrar CDs clássicos antigos, (nós)encontramos uma loja que vendia ec no Iguatemi, mas encontrar CDs clássicos modernos, não encontramos.

$\checkmark \mathrm{EP} \checkmark \mathrm{BP}$

\section{EFEITOS DE CONECTIVIDADE EM PSEUDO-CLIVADAS}

Adaptando estudos clássicos de Akmajian (1970) e Chomsky (1970), Kato et alii (1996) analisaram a estrutura de pseudo-clivadas como sendo derivada de uma estrutura em que a cópula subcategoriza uma mini-oração, na qual o sujeito é o elemento focalizado e o predicado é uma relativa livre.

$$
\operatorname{ser}((\text { você) (quem eu vi)) } \rightarrow \text { Quem eu vi foi você. }
$$

Costa e Duarte (2002) analisam as pseudo-clivadas de modo semelhante, porém, com a diferença de que a relativa livre é o sujeito da mini-oração e o elemento focalizado o predicado.

$$
\text { ser ((quem eu vi) (você)) } \rightarrow \text { Quem eu vi foi você. }
$$

O problema para essas análises é o que vem sendo chamado de problemas de conectividade (Boskovic, 1997), entre eles o problema de a anáfora não ser ccomandada pelo antecedente ${ }^{5}$.

\section{a. O que a Maria é é indigna de si mesma/dela mesma.}

${ }^{5}$ Veja um outro problema de conectividade em pseudo-clivadas do português, visto em Kato e Mioto (2011):

(i) O que cada criança deve trazer é sua (própria) lancheira. 
b. Quem a Maria viu no espelho foi ela mesma.

Boškovič (1997) procura dar uma solução a esse problema assumindo que a FL é o lugar onde a relação de c-comando se estabelece. Partindo da seqüência (14a), o contrapeso (to himself) é movido para dentro da sentença Wh, substituindo what. Este passo crucial é necessário para criar a configuração em que John c-comanda a anáfora himself, figurando como antecedente legítimo para ela:
a. What John is is important to himself.
b. John is important to himself $i_{i}$ ] $t_{i}$

Boeck (s/d) adota uma solução derivacional para o problema do ccomando:
a. John is important.

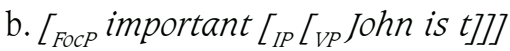
c. [ TopP $\left[J o h n \text { is } t_{i}\right]_{j}\left[_{\text {FocP }}\right.$ important $\left.\left._{i}\left[{ }_{I P}\left[{ }_{V P} t_{j}\right]\right]\right]\right]$
d. [ TopP $\left[\text { What } t_{i} \text { John is } t_{i}\right]_{j}\left[_{\text {FocP }}\right.$ important $\left.\left.\left[_{I P}\left[{ }_{V P} t_{j}\right]\right]\right]\right]$
e. [ TopP $\left[\text { What }{ }_{i} \text { John is } t_{i}\right]_{j}$ is $\left[_{\text {FocP }}\right.$ important $\left.\left.\left[_{I P}\left[{ }_{V P} t_{j}\right]\right]\right]\right]$

Para passar a (15b) important é movido para FocP para checar o traço de foco. Para passar a (15c) o VP [John is $t_{i}$ ] é movido para TopP para checar o traço de tópico. O toque para passar a (15d) consiste em um processo que revive important como o resumptivo what em Spec de TopP para criar a estrutura predicativa presente na relativização. Para obter (15e) a cópula é inserida em Topo para criar a predicação alta, típica das estruturas de tópicocomentário.

Para chegar a uma proposta derivacional alternativa, Kato e Mioto (2011) comparam (16) com (17), mostrando que nelas há efetivamente uma impossibilidade de ligação de de si mesma com Maria por conta da ausência de c-comando. Essa mesma falta de c-comando se observa também nas sentenças simples em (17). Logo, o que se observa em (16) é algum tipo de relação diferente, que precisa ser explicada. 
a. *O que a mãe da Maria ${ }_{i}$ é é indigna de si mesma ${ }_{i}$

b. *Quem a mãe da Maria ${ }_{i}$ viu no espelho foi a si mesma ${ }_{i}$ /ela mesma ${ }_{i}$

a. *A mãe da Maria ${ }_{i}$ é indigna de si mesma ${ }_{i}$.

b. *A mãe da Maria $i$ viu a si mesma ${ }_{i} /$ ela mesma ${ }_{i}$ no espelho.

Kato e Mioto propóem uma alternativa de análise derivacional, abandonando a concepção de que pseudo-clivadas contêm relativas livres, seguindo observação de Hankamer (1974), segundo a qual uma PC não contém uma relativa livre verdadeira. $O$ principal fato que leva o autor a esta conclusão é o fato de que a sentença WH das PCs não pode ser alçada. Resenes (2009), para quem as pseudo-clivadas também não contêm uma relativa, discute o assunto a partir de exemplos como os de (18):
a. * que a Maria é parece ser escandalosa.
b. O que a Maria é parece ser escandaloso.

Em (18b), versão predicacional não-ambígua, a sentença WH pode ser alçada, mas não em (18a). As relativas livres são argumentos que funcionam como DPs e isso condiz com o fato de poderem sofrer alçamento: em (18b) a relativa livre é argumento do predicativo escandaloso e força a concordância de "masculino" no adjetivo. O elemento WH presente numa relativa livre é um operador (portanto, não-anafórico) que liga a posição argumental de onde ele é extraído.

Os problemas de conectividade são solucionados por Kato e Mioto, que assumem a proposta de Kayne (2002), segundo a qual toda relação de construal resulta de movimento sintático. Para a pseudo-clivada (19), teremos a derivação a partir de (20), semelhante à de uma sentença simples, exceto que o DP é complexo.

(19) Quem o João ${ }_{i}$ machucou foi a si mesmo.

(20) a. o joão machucou $_{D P}$ quem $_{i}[$ a si mesmo $\left.i]\right]$

A partir de (20a) o elemento-wh sobe para TopP ou WhP: 
A anáfora a si mesmo do DP se move para FocP, aí interpretado como o foco da pseudo-clivada.

$$
\text { c. } \left.\left[_{F P} \text { a si mesmo }_{i}\left[_{F}\left[{ }_{\text {TopP }}\left[q u e m_{i}\right]\left[_{I P} \text { João machucou }{ }_{D P} t_{i}\left[t_{i}\right]\right]\right]\right]\right]\right]
$$

Nesse ponto insere-se a cópula, que projeta a sentença raiz gerando a pseudo-inversa:

$$
\begin{aligned}
& \text { d. }\left[_ { I P } \text { foi } \left[_ { F P } \text { a si mesmo } { } _ { i } \left[_ { F } L _ { \text { ToppP } } [ q u e m _ { i } ] \left[_ { I P } \text { João machucou } \left[_{S C} t_{i}\right.\right.\right.\right.\right. \\
& \left.\left.\left.\left.\left.\left[t_{i}\right]\right]\right]\right]\right]\right]
\end{aligned}
$$

Há, finalmente, o movimento residual de TopP para o GroundP da sentença raiz, resultando na pseudo-canônica:

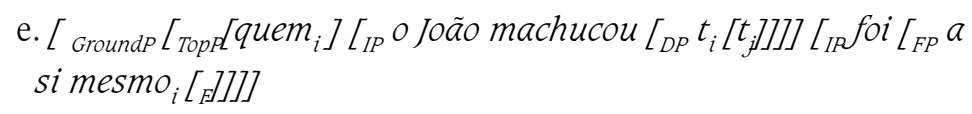

\section{CONCLUSÃo}

Mostrei, neste trabalho, que uma estrutura aparentemente idêntica no $\mathrm{PE}$ e no $\mathrm{PB}$, em que um argumento parece ter sido integralmente topicalizado, na verdade encobre, no PB, um movimento apenas parcial de um DP complexo, que deixa para trás um clítico resumptivo nulo, o que evita que esta variedade do português exiba efeitos de subjacência. Outra construção, que não se limita ao português, a das pseudo-clivadas, levanta o problema da conectividade sem c-comando aparente, quando a derivação pressupõe, como parte da estrutura, uma relativa livre. Utilizando o elemento-Q como parte de um DP-complexo e não como parte de uma relativa livre, o problema da conectividade desaparece. 


\section{REFERÊNCIAS}

AKMAJIAN, A. On deriving cleft sentences from pseudocleft sentences. Linguistic Inquiry, Cambridge, Mass. n. 1, p. 149-168, 1970.

BOECKX, Cedric. Mechanisms of Chain Formation. Ph.D. Dissertation (Doutorado em Linguística) U.Conn, Connecticut, 2001.

BOECKX, Cedric. Pseudoclefts: A Fully Derivational Account. Cambridge: Universidade de Harvard. Ms, (s/d).

BOŠKOVIČ, Željko. Pseudoclefts. Studia Linguistica, Malden, Ma. vol. 51,3 p. 235-277, 1997.

CHOMSKY, Noam. Remarks on nominalization. In: JACOBS, R. A.; ROSENBAUM, P. (eds). Readings in Transformational Grammar. Waltham, Mass.: Ginn, 1970. p. 184-221.

CHOMSKY, Noam. On Wh-Movement. In: CULICOVER, P.; WASOW, T.; AKMAJIAN, A. (eds). Formal Syntax. New York: Academic Press, 1977.

CINQUE, Gugliemo. Types of A'-Dependencies. Cambridge, Mass: MIT Press, 1990.

FARRELL, Patrick. Null Objects in Brazilian Portuguese. NLLT, Dordrecht. volume 8, 2. p. 325346, 1990.

COSTA, João e DUARTE, Inês. Minimizando a estrutura: uma análise unificada das construções de clivagem em português. Actas da APL, Lisboa, 2002. p. 627-638.

GALVES, Charlotte. O Objeto Nulo no Português Brasileiro; percurso de uma pesquisa. Caderno de Estudos Lingüísticos, Campinas .vol. 17, p. 65-90, 1989.

HANKAMMER, Jorge. On the noncyclic nature of wh-clefting. Proceedings of CLS, Chicago, Il, n. 10, p. 221-233, 1974.

HORNSTEIn, Norbert. Move! A Minimalist Theoy of Construal. Oxford: Blackwell, 2000.

KATO, Mary. A. The distribution of null and pronominal objects in Brazilian Portuguese. Linguistic Perspectives on the Romance Languages: Selected Papers from the XXI Linguistic Symposium on Romance Languages (Currents Issues in Linguistic Theory Series). Amsterdam: John Benjamins, 1993. p. 225-235.

; Braga, M. Luiza; Corrêa, Vilma C.; Rossi, M.A. L e Sikansi,N.S. (eds) Asconstruções-Q no português brasileiro falado: perguntas, clivadas e relativas. In: Koch, I.G.V. (ed.) Gramática do Português Falado, Vol VI: Desenvolvimentos. Campinas: Editora da UNICAMP. 1996. p. 303-370

; RAPOSO, Eduardo. Topicalization in European and Brazilian Portuguese. In: CAMACHO, J; CABRERA, M. J.; SÁNCHEZ, L.; DEPREZ, V. (eds). Romance Linguistics: selected papers from the 36th Linguistic Symposium on Romance Languages. Amsterdam: John Benjamins, 2007. 
LSRL 213-226.

; MIOTO, Carlos. Pseudo clivadas e os efeitos de conectividade. In: Estudos Formais da Gramática das Línguas Naturais - Anais do Encontro Nacional do Grupo de Trabalho Teoria da Gramática. Goiânia: Editora Cânone, 2011. p. 51-66.

KAYNE, Richard. Pronouns and their antecedents. In: EPSTEIN, S.; SEELY, D. (eds). Derivation and Explanation in the Minimalist Program. Malden, Blackwell, 2001. p. 133-166.

MATOS, M. Gabriela. Construções de Elipse de Predicado em Português: SV Nulo e Despojamento. Dissertação de Doutorado em Linguística- Faculdade de Letras, Universidade de Lisboa, 1992.

RAPOSO, Eduardo. The null object in European Portuguese. In: JAEGGLI, O.; SILVA-CORVALÁN, C. (eds). Studies in Romance Linguistics. Dordrecht: Foris, 1986. p. 373-390.

. Definite/Zero Alternations in Portuguese: Towards a Unification of Topic Constructions. In: SCHWEGLER, A.; TRANEL, B.; URIBE-ETXEBARRIA, M. (eds). Romance Linguistics: Theoretical Perspectives. Amsterdam: John Benjamins Co., 1996. p. 197-212.

RESENES, Mariana. S. de. Sentenças pseudo-clivadas do português brasileiro. 143fls.. Mestrado em Linguística - Faculdade de Letras, UFSC, Florianópolis, 2009.

URIAGEREKA, Juan. Aspects of the Syntax of Clitic Placement in Western Romance. Linguistic Inquiry, Cambrige, Mass. Vol. 26, 1. p. 79-123, 1995.

Submetido em: 28/11/2011

Aceito em: 17/01/2012 
